\title{
Cascaded PID controller for anaesthesia delivery
}

\author{
Alena Simalatsar ${ }^{1}$, Monia Guidi ${ }^{23}$, and Thierry Buclin ${ }^{2}$ \\ Emails: alena.simalatsar@epfl.ch,monia.guidi@chuv.ch,thierry.buclin@chuv.ch \\ ${ }^{1}$ Ecole Polytechnique Fédérale de Lausanne (EPFL), Lausanne, Switzerland \\ 2 Division of Clinical Pharmacology Centre Hospitalier Universitaire Vaudois (CHUV), Lausanne, Switzerland \\ ${ }^{3}$ School of Pharmaceutical Sciences, University of Geneva, University of Lausanne, Geneva, Switzerland
}

\begin{abstract}
The technologies for continuous measurement of the anaesthetic agents circulating in body fluids are not mature yet, though some preliminary prototypes exist already. We present a control algorithm that based on the real measurement of propofol plasma concentration may adjust the delivery rate. This opens a possibility for a safer anesthesia when the technologies for online measurement of drug concentration will be mature enough to be combined with our model.
\end{abstract}

\section{INTRODUCTION}

The controlled delivery of intravenous anesthetics aims at fast and safe achievement and maintenance of a certain depth of hypnosis (DOH), correlated with the plasma concentration of the administered drugs (e.g. propofol, fentanyl or midazolam, etc.). Today, such drugs are regularly injected by Target Controlled Infusion (TCI) systems, piloted by quantitative predictions of plasma drug concentration based on pharmacokinetic (PK) models complemented with an algorithm, that based on the predicted output (e.g. drug concentration) adjusts the drug delivery rate in an open-loop manner [11]. However, the inaccuracy of these models can reach up to 30$50 \%$ [1] due to inter- and intra patients' variability. Instead of relying on drug concentration in plasma, some attempts to improve the situation where made by introducing a closedloop control that uses the electroencephalogram (EEG) monitoring to derive the bispectral index (BIS) associated to the DOH [4]. However, BIS only indirectly depends on drug delivery rate, is delayed with respect to the changes of drug concentration in plasma, and can be affected by various sources of noise, which can lead to overdosing or unexpected awakening of a patient.

The situation can be improved by developing sensors for continuous drug measurement in the blood that would be the basis for a new closed-loop control. The classical closed-loop controlling algorithm is the Proportional-Integral-Derivative (PID) algorithm that is used to minimise the error of the output variable of the physical part by computing the input value as a weighted sum of this error. The error is computed as a difference between the real measured and desired level of the variable. The real measurement of the concentration of anesthetic agents circulating in body fluids was not possible until now, since there are no mature technologies yet, though some preliminary prototypes exist already [3], [2]. However, even when the technology will be mature enough, such measurements will be affected by measurement noise and will be delayed with respect to patients response. Therefore, they will help only in narrowing down the inaccuracy of the PK models. The further improvement will be made by performing controlled delivery of anesthetics based on both plasma drug concentration and $\mathrm{DOH}$.

In this paper we present a closed-loop control algorithm for the delivery of propofol, an anesthetic widely used for general anesthesia. Currently, instead of the estimated plasma concentration corrected with real measurements we directly use the concentration values predicted by the population PK and pharmacodynamic (PD) models [8] respectively. In particular, in this paper we employ the Schnider [6], [7] PK model, which allows us to compare the performance of our closed-loop approach with the performance of the existing Orchestra ${ }^{\circledR}$ BasePrimea TCI pump from Fresenius, widely used in hospitals for general anesthesia delivery. Potentially, the algorithm may simply control the plasma concentration; it can also control the drug concentration at the effect site, which in case of anesthetics is the brain, while keeping plasma concentration within a limit. We present a cascaded PID control algorithm that allows fast and safe achievement of the target values of the effect site concentration while keeping the plasma concentration within a limit. Nevertheless, it can be easily extended for the control of the level of $\mathrm{DOH}$. The performance of the algorithm when controlling the effect site concentration is verified against the data from the Orchestra ${ }^{\circledR}$ Base Primea TCI system from Fresenius.

The paper is organised as follows. Section II presents the background on existing TCI pumps. Section III describes the PK-PD models of propofol. In section IV we present the control algorithm and evaluate its performance in section $\mathrm{V}$.

\section{TARget Controlled Infusion of AnAesthesia}

Anesthetics are usually short-time acting, intravenously administered drugs. They require an ensured multi-step gradation of the effect intensity provided by the drug concentration in the blood. Therefore, anesthesiologists need to continuously adjust the delivered rate in order to keep the drug concentration at a certain level for an appropriate degree of sedation and avoid both patient's intoxication and awareness. Propofol is a widely used anesthetic agent, therefore the achievement and maintenance of its particular concentration is of particular interest. Several software tools computing the required delivery rate based on changes of propofol concentration in blood has been developed in the 
past: STANPUMP [10], IVASIM [9] and RUGLOOP [12]. Some of these algorithms have been commercialised.

One of the widely used TCI system for the delivery of anesthetics and analgesics is the Orchestra ${ }^{\circledR}$ Base Primea system from Fresenius. It consists of several identical modules, each one is designed to deliver: propofol, remifentanil or sufentanil often given in cocktail during the surgery. The independent modules of the TCI pump can control the drug infusion based either on the plasma or effect site concentration that are predicted with one of the offered PK models. For propofol delivery it is possible to choose either Marsh [5] or Schnider [6], [7] models. While the parameters of the PK models are openly available, the precise mechanism of controlling the drug delivery rate is not presented in the documentation but is a proprietary implementation of the software tools mentioned above.

\section{PK-PD MODELING}

The PK of propofol is usually described by a three compartment model extended with a virtual compartment representing the effect site, which for anesthetic drug is the brain. The PK-PD model for propofol is presented on Figure 1. On the figure, the compartment with the volume $V_{1}$ is the central compartment and represents the plasma of the patient. The drug is delivered to the central compartment with the delivery rate $U$ and is cleared from it with elimination rate $k_{10}$. The two peripheral compartments with volumes $V_{2}$ and $V_{3}$ are the shallow and the deep compartments needed to model the drug distribution and its further release into/by various tissues, characterised by coefficients $k_{12}, k_{21}, k_{13}$ and $k_{31}$. The system of differential equations 1,2 and 3 describes the change of drug concentrations in the three compartments:

$$
\begin{aligned}
\frac{d C_{1}}{d t}=C_{2} k_{21}+C_{3} k_{31}-C_{1}\left(k_{10}+k_{12}+k_{13}\right)+U \\
\frac{d C_{2}}{d t}=C_{1} k_{12}-C_{2} k_{21} \\
\frac{d C_{3}}{d t}=C_{1} k_{13}-C_{3} k_{31}
\end{aligned}
$$

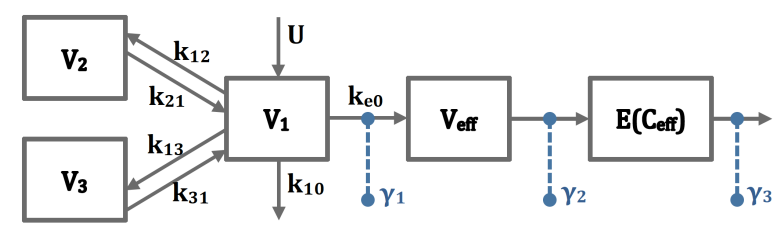

Fig. 1: 3 compartment PK-PD model

The estimated concentration of the drug at the effect site, marked as a virtual compartment with the volume $V_{\text {eff }}$, is computed from the concentration in the central compartment (transfer rate $k_{e 0}$ ) described by Eq. 4 :

$$
\frac{d C_{e f f}}{d t}=\left(C_{1}-C_{e f f}\right) k_{e 0}
$$

It must be noted that the virtual compartment does not affect the concentration in the central compartments, such that there is not absorption or further release of the drug from/into plasma. In this paper we will be using the parameters of the Schnider model [6], [7].

The most right block on Figure 1 represents the PD part of the model where $E\left(C_{e f f}\right)$ stands for the level of the effect that is the function of the effect site concentration $C_{e f f}$. $E\left(C_{e f f}\right)$ is usually described with a Hill sigmoid [8]:

$$
E\left(C_{e f f}\right)=E_{0}+\left(1-E_{0}\right) \frac{C_{e f f}^{\gamma}}{C_{e f f, 50}^{\gamma}+C_{e f f}^{\gamma}}
$$

where the value $E=E_{0} \approx 0$ corresponds to the fully awake state and $E=1$ to total absence of cortical activity, $C_{\text {eff } f, 50}$ is the $C_{\text {eff }}$ values that corresponds to $50 \%$ clinical effect and $\gamma>1$ is a parameter determining the shape of the sigmoid.

\section{Control Algorithm}

The controlled delivery of anaesthetics in general requires the fast and safe achievement of certain $\mathrm{DOH}$ that depends on the effect site concentration $C_{e f f}$ that transitively depends on the plasma concentration of the drug $C_{1}$. For the purposes of the control problem presented further we will consider three different scenarios for a control algorithm: (1) control of only the plasma concentration $C_{1}$ (control point $\gamma_{1}$ on Figure 1); (2) control of the effect site concentration $C_{e f f}$, while keeping the plasma concentration within a limit (control point $\gamma_{2}$ ); (3) control the BIS index while keeping the plasma concentration within a limit (control point $\gamma_{3}$ ). We propose to use a classical PID controller for the above scenarios.

The currently existing TCI pumps, such as BasePrimea from Fresenius, allow two modes of delivery, (1) and (2), which we evaluate against our control algorithm in Section V.

\section{A. Classical PID controller}

The PID control algorithm is used to minimise the error $e$ of the output variable of a physical system by computing the input value $I$ as a weighted sum of this error. The error value is computed as a difference between the actual measured output variable and desired level of that variable. For digital implementation of the controller the weighted sum is computed at every sampling instance $t_{k}$ as follows:

$$
I\left(t_{k}\right)=K_{p} * e\left(t_{k-1}\right)+K_{i} * e_{i}\left(t_{k-1}\right)+K_{d} * e_{d}\left(t_{k-1}\right)
$$

where $K_{p}, K_{i}, K_{d}$ and $e, e_{i}$, and $e_{d}$ are the proportional, integral and derivative coefficients and errors respectively. The error $e_{p}$ is simply the error of the output variable. The computation for the derivative error $e_{d}$ requires the implementation of the low pass filter to reduce the sensitivity of the derivative term to random changes:

$$
e_{d}\left(t_{k}\right)=\frac{T_{d}}{T_{d}+N \Delta t}\left[e_{d}\left(t_{k-1}\right)-K_{p} N\left(e\left(t_{k}\right)-e\left(t_{k-1}\right)\right)\right]
$$


where $N$ is the filter order, $\Delta t=t_{k}-t_{k-1}$ and $T_{d}=K_{d} / K_{p}$. The integral error is computed as follows:

$$
e_{i}\left(t_{k+1}\right)=e_{i}\left(t_{k}\right)+\frac{K_{p} \Delta t}{T_{i}} e\left(t_{k}\right)+\frac{\Delta t}{T_{f}} e_{s}
$$

where the second term of the equation is used to memorise the changes of the error $e$, while the third term is used to prevent integration wind-up in PID controllers when the actuator cannot provide the value $v$ computed by the controller due to the actuator physical limit at value $u$. This way, $e_{s}=v-u$, while the integrator reset time $T_{f}$ is chosen between $T_{i}$ and $T_{d}$, where $T_{i}=K_{p} / K_{i}$ and $T_{d}=K_{d} / K_{p}$. In this paper we choose the following value: $T_{f}=\left(T_{i}+T_{d}\right) / 2$.

\section{B. Cascaded PID control}

In case of scenario (1), when targeting the $C_{1}$ concentration, it is enough to introduce one PID controller that based on the error $e=$ target $_{C_{1}}-C_{1}$ will compute the required delivery rate $U$. The second and the third scenarios are more complicated: fast achievement of $C_{e f f}$ or DOH requires high concentration $C_{1}$, which at the same time, can not exceed a certain level. Therefore, in order to be able to control two points $\gamma_{1}$ and $\gamma_{2}$, or $\gamma_{1}$ and $\gamma_{3}$, we propose to use a cascaded system of two PID controllers as shown on Figure 2.

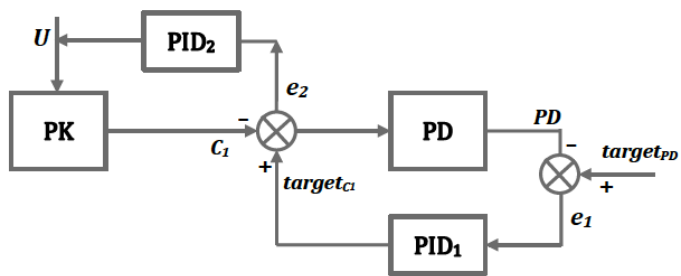

Fig. 2: Cascaded PID control of PK-PD model

Essentially, the output of the first controller $P I D_{1}$ is the target concentration in the central compartment target $_{C_{1}}$ needed for fast and safe achievement of the target value at the effect site $\operatorname{target}_{P D}$ based on the error $e_{1}=\operatorname{target}_{P D}-$ $P D$. The output variable $P D$ and its target value $\operatorname{target}_{P D}$ can be either the predicted concentration at the effect site $C_{e f f}$ and its target values target $_{C_{e f f}}$ or the predicted effect $E\left(C_{e f f}\right)$ and the desired DOH level. The $P I D_{2}$ controller, in turn, will be computing the actual drug delivery rate $U$ for fast and safe achievement of target $_{C_{1}}$ value based on the error $e_{2}=$ target $_{C_{1}}-C_{1}$. Note that target $_{C_{1}}$ is dynamically changing. Such a structure allows a fast achievement of the target concentration at the effect site while controlling the drug concentration in plasma, so that it does not go over certain level, which for Base Primea TCI pump is $15(\mathrm{mg} / \mathrm{L})$. Further we will present the case of scenario (2).

\section{Tuning cascaded PID controller}

It must be noted that we have more liberty in choosing the coefficients of the external controller $P I D_{2}$ because there is less restriction on safe achievement of the target value and the physical system under control is described with a system of differential equations 1-3 making it reluctant to input changes. Thus, we choose the following coefficients: $K_{p_{2}}=$ $100, K_{i_{2}}=0.13$, and $K_{d_{2}}=0.003$. The value controlled by the $P I D_{2}$ is the system input $U$ that has upper and lower limits for the delivery rate, $0<=U_{\text {lim }}<=200 \mathrm{mg} / \mathrm{min}$. When any of the limits of the delivery rate is reached, the third term of Eq. 8 will be computed with $e_{s}=U-U_{\text {lim }}$.

The internal controller $P I D_{1}$ of the cascade is much more sensitive to parameter changes than the external one, where the values of the proportional and integral coefficients are playing a crucial role in controller performance and stability. Both coefficients are directly proportional with the target values $\operatorname{target}_{P D}$. It must also be noted that the $\operatorname{target}_{P D}$ value may be changed during the infusion. The difference between the new and the old target values $J$ is inversely proportional with the coefficients of $P I D_{1}$. We have developed a semiautomatic coefficients tuning by providing a table of $K_{p_{1}}$ and $K_{i_{1}}$ values for ranges of target $_{P D}$ and $J$.

We must also take into account that the input target $_{C_{1}}$ computed by $P I D_{1}$ is the concentration of the central compartment $C_{1}$ that is limited $0<=C_{\text {lim }}<=15 \mathrm{mg} / \mathrm{l}$. When the upper limit is reached, we have to maintain the concentration of the central compartment at this limit until the computed value of target $_{C_{1}}$ does not drop below this limit. To achieve that, the delivery rate $U$ of the drug (input value of the $P I D_{2}$ controller) should be set to the value that will maintain the current maximum allowed concentration of the central compartment, meaning that $d C_{1} / d t=0$ :

$$
U=-C_{2} k_{21}-C_{3} k_{31}+C_{1}\left(k_{10}+k_{12}+k_{13}\right)
$$

where the values of $C_{1}, C_{2}$ and $C_{3}$ are the drug concentration values when the limit of $C_{1}$ is achieved.

Also the value target $_{C_{1}}$ can not be immediately provided, since the plasma concentration can be only gradually achieved with a speed described by the PK model. We must compensate the integral term according to the difference between the value computed by the controller and the limit value using $e_{s}=$ target $_{C_{1}}-C_{1}$ for the third term of Eq. 8 .

The target concentration may be changed during the operation which will require the change of coefficients of $P I D_{1}$. For the bumpless parameters change the integral term of $P I D_{1}$ controller at each change must be adjusted as follows:

$$
\begin{gathered}
e_{i}^{\text {new }}=e_{i}^{\text {old }}+\left(K_{p}^{\text {old }}-K_{p}^{\text {new }}\right)\left(\text { target }_{P D}-P D\right) \\
\text { V. Evaluation }
\end{gathered}
$$

\section{Evaluation}

To validate our model we have performed several experiments with the Base Primea pump without involving any patient. We configured the pump with the parameters for 4 different imaginary patients: (1) a male of 36 years old, with body weight $70 \mathrm{~kg}$ and height $170 \mathrm{~cm}$; (2) a female of 25 years old with body weight $50 \mathrm{~kg}$ and height $150 \mathrm{~cm}$; (3) a male of 36 years old, with body weight $90 \mathrm{~kg}$ and height $170 \mathrm{~cm}$; and (4) a male of 65 years old, with body weight $90 \mathrm{~kg}$ and height $170 \mathrm{~cm}$. For each patient we have performed 3 experiments by changing targets for the drug concentrations at the effect site $C_{\text {eff }}$ to: (exp. 1) 6, 4, 5 and then to $0 \mathrm{mg} / l$; (exp. 2) $2,3,4,5$ and then to $0 \mathrm{mg} / \mathrm{l}$; (exp. 
3) 2, 4, 6 and then to $0 \mathrm{mg} / \mathrm{l}$. The TCI data, i.e. the predicted drug concentration for plasma and effect site and the delivery rates, were loaded to the computer. Thus we could compare them to the performance of our algorithm configured with identical patient parameters and changes of target values.

Figure 3 presents the comparison of the results from our algorithm with the values from TCI for the first patient with the first experimental settings. The continuous lines represent the values computed by our controller while the dots correspond to the data collected from TCI pump. We can see a good accordance between our algorithm and the data of the TCI. Only when the target is decreased does our model show more caution with respect to TCI.

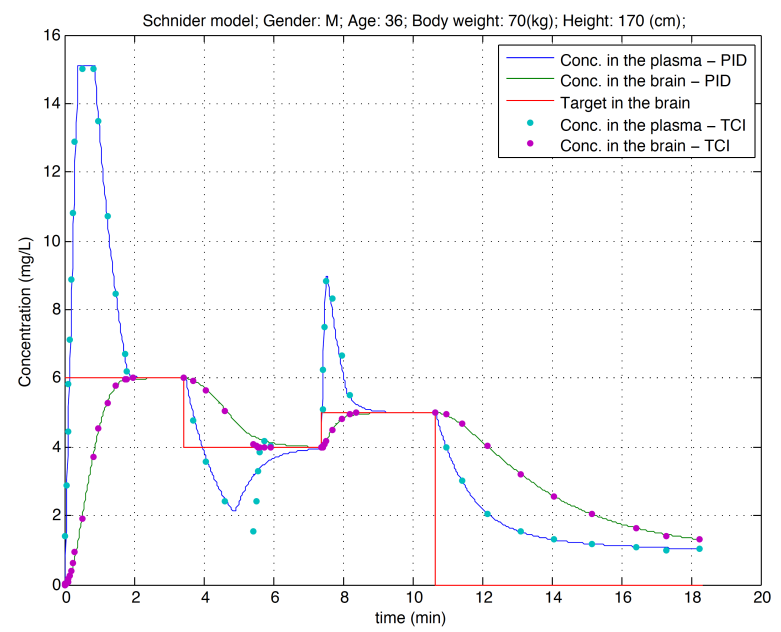

Fig. 3: Achievement of target $_{C_{e f f}}$ : Exp 1, Patient 1.

Figure 4 presents the comparison of the delivery rates ( $\mathrm{mg} / \mathrm{min}$, divided by 20 to better fit the graph) computed by our model. It can be seen that our model computes the delivery rates for initial shots higher than the TCI. However, this shots are shorter in time compared to the TCI, which makes the total amount of the drug delivered identical.

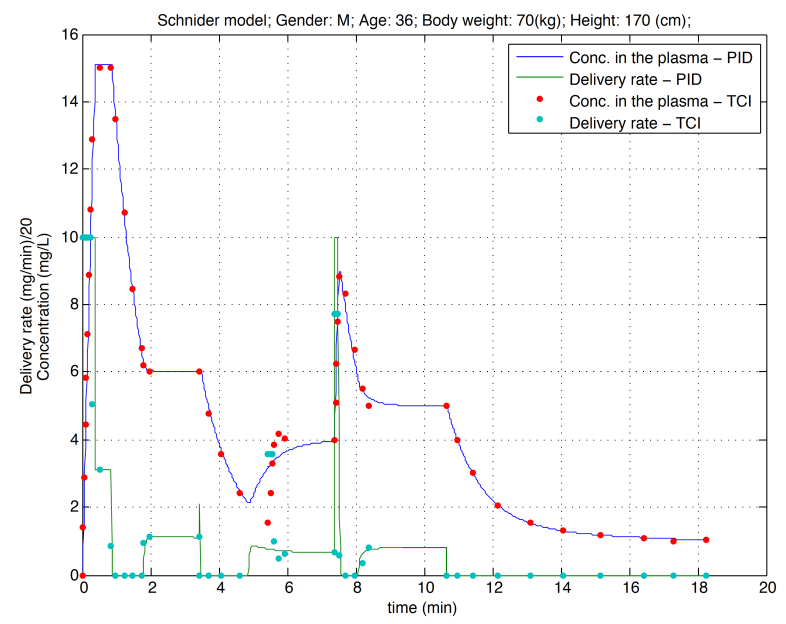

Fig. 4: Achievement of target $_{C_{e f f}}$ : Exp 1, Patient 1.

\section{CONCLUSION}

The existing open-loop solution can potentially adapt the Bayesian approach for run-time individualisation of PK-PD model parameters, with each new measurement. However, this approach is not dealing with measurement noise, which in case of BIS index can be quite large. The incorporation of the PK model and real measurements is possible using Kalman filter, which can be tuned to place either more weight on the measurement or on the PK-PD model. Since, it is not suitable to improve the existing open-loop solution, the implementation of the closed-loop controller is required. Therefore, the closed-loop control algorithm presented in this paper represents a first step towards improvement of precision of the TCI-based anesthesia. We choose the PID algorithm rather than any higher order linear controller since it is easier to tune and it already shows a good accordance with the data from Base Primea TCI system. The cascaded version provides modularity allowing to compose a specific controller for each scenario described in Section IV. We have run the experiments for 4 patients with parameter settings covering extreme cases, provoking maximum variability in PK model dynamics and our algorithm showed good stability. Proving the algorithm robustness with more extreme cases is certainly the next step in our future work.

\section{ACKNOWLEDGMENT}

The research work presented in this paper is funded by the CoMofA Project of Swiss NSF foundation.

\section{REFERENCES}

[1] D. Eleveld, J. Proost, L. Cortinez, A. Absalom, and M. Struys. A general purpose pharmacokinetic model for propofol. Anesthesia and analgesia, 118(6):1221-1237, 2011

[2] F. Kivlehan, F. Garay, J. Guo, E. Chaum, and E. Lindner. Toward feedback-controlled anesthesia: voltammetric measurement of propofol (2,6-diisopropylphenol) in serum-like electrolyte solutions. Analytica Chimica Acta, 84(18):7670-7676, 2012.

[3] J. Langmaier, F. Garay, F. Kivlehan, E. Chaum, and E. Lindner. Electrochemical quantification of 2,6-diisopropylphenol (propofol). Analytica Chimica Acta, 704(1-2):63 - 67, 2011.

[4] N. Liu, T. Chazot, S. Hamada, A. Landais, N. Boichut, C. Dussaussoy, B. Trillat, L. Beydon, E. Samain, D. I. Sessler, and M. Fischler Closed-loop coadministration of propofol and remifentanil guided by bispectral index: a randomized multicenter study. Anesthesia and analgesia, 12(3):546-557, 2011.

[5] B. Marsh, M. White, N. Morton, and G. N. C. Kenny. Pharmacokinetic model driven infusion of propofol in children. British Journal of Anaesthesia, 67(1):41-48, 1991.

[6] T. Schnider, C. Minto, P. L. Gambus, C. Andresen, D. Goodale, S. Shafer, and E. Youngs. The influence of method of administration and covariates on the pharmacokinetics of propofol in adult volunteers. Anesthesiology, 88(5):1170-1182, 1998.

[7] T. W. Schnider, C. F. Minto, S. L. Shafer, P. L. Gambus, C. Andresen, D. B. Goodale, and E. J. Youngs. The influence of age on propofol pharmacodynamics. Anesthesiology, 90(6):1502-1516., 1999.

[8] T. W. Schnider, DrMed, C. C. F. Minto, MB, S. L. Shafer, MD, P. L. Gambus, MD, C. Andresen, MD, P. D. B. Goodale, DDS, and E. J. Youngs, MD. The influence of age on propofol pharmacodynamics. Anesthesiology, 90(6):1502-1516, 1999.

[9] J. Schüttler and S. Kloos. IVASIM. 1991

[10] S. Shafer. STANPUMP User's manual. 1999.

[11] S. L. Shafer and K. M. Gregg. Algorithms to rapidly achieve and maintain stable drug concentrations at the site of drug effect with a computer-controlled infusion pump. Journal of Pharmacokinetics and Biopharmaceutics, 20(2):147-169, 1992.

[12] T. D. Smet and M. Struys. RUGLOOP. 2001. 